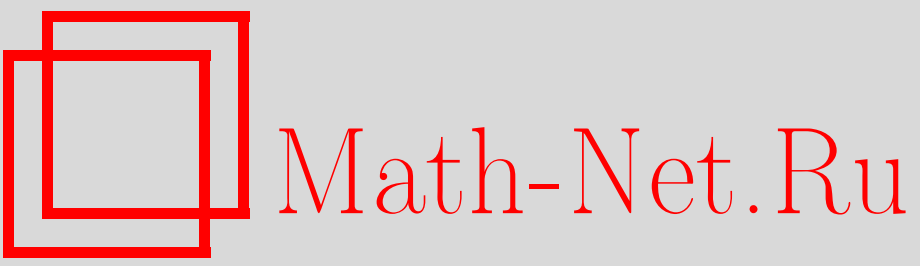

Д. Байатт-Смит, Х. У. Браден, О функциональном уравнении Руджинарса, ТМФ, 2002, том 133, номер 3, 353-366

DOI: https://doi.org/10.4213/tmf403

Использование Общероссийского математического портала Math-Net.Ru подразумевает, что вы прочитали и согласны с пользовательским соглашением

http://www.mathnet.ru/rus/agreement

Параметры загрузки:

IP: 54.81 .137 .203

26 апреля 2023 г., 14:27:40 
ТЕОРЕТИЧЕСКАЯ

И МАТЕМАТИЧЕСКАЯ

ФИЗИКА

Том 133, № 3

декабрь, 2002

(C) 2002 г. Дж. Г.Б. Байатт-Смит*, Х.У. Браден*

\section{О ФУНКЦИОНАЛЬНОМ УРАВНЕНИИ РУДЖИНАРСА}

Получено общее решение функционального уравнения, введенного Руджинарсом, которое гарантирует коммутативность $n$ операторов, связанных с квантовыми моделями Руджинарса-Шнайдера.

Ключевые слова: интегрируемость, функциональные уравнения.

\section{1. ВВЕДЕНИЕ}

Целью настоящей работы является исследование функционального уравнения

$$
\sum_{\substack{I \subseteq\{1,2, \ldots, n\} \\|I|=k}}\left(\prod_{\substack{i \in I \\ j \notin I}} h\left(x_{j}-x_{i}\right) h\left(x_{i}-x_{j}-i \beta\right)-\prod_{\substack{i \in I \\ j \notin I}} h\left(x_{i}-x_{j}\right) h\left(x_{j}-x_{i}-i \beta\right)\right)=0, \quad(1)
$$

где $\beta$ - произвольное положительное число, а сумма берется по всем подмножествам с $k$ элементами. Мы покажем, что это уравнение лежит в основе квантовой интегрируемости моделей Руджинарса-Шнайдера. Докажем, что справедлива следующая

ТЕОРема 1. Общее решение функционального уравнения (1), аналитичное в окрестности вещественной оси с простым полюсом в начале координат или с набором таких полюсов в точках пр на вещественной оси $(n \in \mathbb{Z})$, имеет вид

$$
h(x)=b \frac{\sigma(x+\nu)}{\sigma(x) \sigma(\nu)} e^{\alpha x}
$$

Преж де чем приступить к доказательству теоремы 1, очертим контекст данной работы. Несколько лет назад Руджинарс и Шнайдер [1] провели исследование механических моделей, подчиняюшихся алгебре Пуанкаре

$$
\{H, B\}=P, \quad\{P, B\}=H, \quad\{H, P\}=0,
$$

*Department of Mathematics and Statistics, The University of Edinburgh, Edinburgh, UK. E-mail: Byatt@ed.ac.uk; hwb@ed.ac.uk 
где $H$ - гамильтониан системы, генерирующий временны́е трансляции, $P$ - генератор пространственных трансляций, а $B$ - генератор бустов. Это исследование было частично мотивировано поиском механических моделей, описывающих взаимодействие солитонов. Оказалось, что модели, которые они открыли, обладают другими важными свойствами: они в действительности являются интегрируемыми и существует их естественная квантовая версия. Руджинарс и Шнайдер приняли анзац

$$
H=\sum_{j=1}^{n} \operatorname{ch} p_{j} \prod_{k \neq j} f\left(x_{j}-x_{k}\right), \quad P=\sum_{j=1}^{n} \operatorname{sh} p_{j} \prod_{k \neq j} f\left(x_{j}-x_{k}\right), \quad B=\sum_{j=1}^{n} x_{j} .
$$

При использовании канонической скобки Пуассона $\left\{p_{i}, x_{j}\right\}=\delta_{i j}$ в рамках этого анзаца автоматически удовлетворяются первые два соотношения в (3), включающие оператор бустов $B$. Последняя в (3) скобка Пуассона тогда равна

$$
\begin{aligned}
& \{H, P\}=-\sum_{j=1}^{n} \partial_{j} \prod_{k \neq j} f^{2}\left(x_{j}-x_{k}\right)- \\
& -\frac{1}{2} \sum_{j \neq k} \operatorname{ch}\left(p_{j}-p_{k}\right) \prod_{l \neq j} f\left(x_{j}-x_{l}\right) \prod_{m \neq k} f\left(x_{k}-x_{m}\right)\left(\partial_{j} \ln f\left(x_{k}-x_{j}\right)+\partial_{k} \ln f\left(x_{j}-x_{k}\right)\right),
\end{aligned}
$$

и для того чтобы независимые члены, пропорциональные $\operatorname{ch}\left(p_{j}-p_{k}\right)$, обращались в нуль, требуется, чтобы отношение $f^{\prime}(x) / f(x)$ было нечетным. Отсюда вытекает, что $f(x)$ является или четной, или нечетной функцией ${ }^{1)}$, и в любом случае функция $F(x)=f^{2}(x)$ четна. Если $f(x)$ удовлетворяет таким условиям, окончательная скобка Пуассона эквивалентна функциональному уравнению:

$$
\{H, P\}=0 \Longleftrightarrow \sum_{j=1}^{n} \partial_{j} \prod_{k \neq j} f^{2}\left(x_{j}-x_{k}\right)=0
$$

Заметим, что поделив уравнение (1) на $\beta$ и взяв предел при $\beta \rightarrow 0$, получим (4), где $F(x)=h(x) h(-x)$ при $k=1$.

При $n=3$ уравнение (4) можно записать в виде

$$
\left|\begin{array}{ccc}
1 & 1 & 1 \\
F(x) & F(y) & F(z) \\
F^{\prime}(x) & F^{\prime}(y) & F^{\prime}(z)
\end{array}\right|=0, \quad x+y+z=0
$$

где $F(x)=f^{2}(x)$. Руджинарс и Шнайдер показали [1], что $F(x)=\wp(x)+c$ удовлетворяет уравнению (5) и, более того, удовлетворяет уравнению (4) для всех $n$. То же самое функциональное уравнение (без допущения о четности функции $F(x)$ ) возникало в нескольких разных контекстах, связанных с интегрируемыми системами. Оно появляется при характеризации квантово-механических потенциалов, для которых волновая

\footnotetext{
${ }^{1)}$ Руджинарс и Шнайдер полагают $f(x)=f(-x)$.
} 
функция основного состояния (заданного вида) факторизуема [2] $]^{2)}$. Недавно было показано [3], что это уравнение характеризует систему Калоджеро-Мозера [4], которая представляет собой скейлинговый предел системы Руджинарса-Шнайдера [5]. Возникают глубокие связи между функциональными уравнениями и интегрируемыми системами [6]. Аналитические решения уравнения (5) были охарактеризованы Бухштабером и Переломовым [7], а затем нами был получен [8] несколько более сильный результат с использованием значительно более простого доказательства.

Теорема 2 [8]. Пусть $F$ - трижды дифференцируемая функция, удовлетворяющая функииональному уравнению (5). Тогда с точностью до явной инвариантности $F(z) \rightarrow \alpha F(\delta z)+\beta$ одна из функиий $F(z)=\wp(z+d), \quad F(z)=e^{z}$ или $F(z)=z$

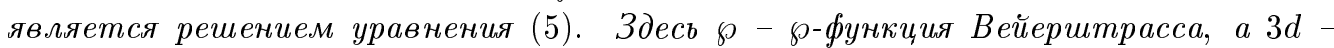
точка решетки для ю-функции.

Таким образом, четные решения уравнения (5) являются в точности решениями, полученными Руджинарсом и Шнайдером. До самого последнего времени обшее решение уравнения (4) оставалось неизвестным, в работе [9] мы установили следуюшую теорему.

Теорема 3 [9]. Общее четное решение уравнения (4) в классе мероморфных функиий, единственные сингулярности которых на вещественной оси являются или двойным полюсом в начале координат, или двойными полюсами в точках $n p(p \in \mathbb{R}, n \in \mathbb{Z})$, для всех нечетных $n$ дается решением Руджинарса-Шнайдера а для четньх $n \geqslant 4$, в дополнение к решениям Руджинарса-Шнайдера, имеются следующие решения:

$$
\begin{aligned}
F_{1}(z) & =\sqrt{\left(\wp(z)-e_{2}\right)\left(\wp(z)-e_{3}\right)}=\frac{\sigma_{2}(z) \sigma_{3}(z)}{\sigma^{2}(z)}= \\
& =\frac{\theta_{3}(v) \theta_{4}(v)}{\theta_{1}^{2}(v)} \frac{\theta_{1}^{\prime 2}(0)}{4 \omega^{2} \theta_{3}(0) \theta_{4}(0)}=b \frac{\operatorname{dn} u}{\operatorname{sn}^{2} u}, \\
F_{2}(z) & =\sqrt{\left(\wp(z)-e_{1}\right)\left(\wp(z)-e_{3}\right)}=\frac{\sigma_{1}(z) \sigma_{3}(z)}{\sigma^{2}(z)}= \\
& =\frac{\theta_{2}(v) \theta_{4}(v)}{\theta_{1}^{2}(v)} \frac{\theta_{1}^{\prime 2}(0)}{4 \omega^{2} \theta_{2}(0) \theta_{4}(0)}=b \frac{\operatorname{cn} u}{\operatorname{sn}^{2} u}, \\
F_{3}(z) & =\sqrt{\left(\wp(z)-e_{1}\right)\left(\wp(z)-e_{2}\right)}=\frac{\sigma_{1}(z) \sigma_{2}(z)}{\sigma^{2}(z)}= \\
& =\frac{\theta_{2}(v) \theta_{3}(v)}{\theta_{1}^{2}(v)} \frac{\theta_{1}^{\prime 2}(0)}{4 \omega^{2} \theta_{2}(0) \theta_{3}(0)}=b \frac{\mathrm{cn} u \operatorname{dn} u_{\operatorname{sn}^{2} u}}{}
\end{aligned}
$$

Здесь

$$
\sigma_{\alpha}(z)=\frac{\sigma\left(z+\omega_{\alpha}\right)}{\sigma\left(\omega_{\alpha}\right)} e^{-z \zeta\left(\omega_{\alpha}\right)}, \quad u=\sqrt{e_{1}-e_{3}} z, \quad v=\frac{z}{2 \omega}, \quad b=e_{1}-e_{3},
$$

2) Заметим, что $\delta$-функциональный потенциал $a \delta(x)$ в квантово-механической задаче многих тел на прямой, имеющей факторизуемую волновую функцию основного состояния, можно рассматривать как предел $-b /\left(\alpha \operatorname{sh}^{2}(-x / \alpha+\pi i / 3)\right)$ при $\alpha \rightarrow 0$, где $\pi a \alpha=6 b$. Таким образом, все известные квантово-механические задачи с факторизуемой волновой функцией основного состояния включаются в уравнение (5). 
причем $\omega_{1}=\omega, \omega_{2}=-\omega-\omega^{\prime}$ и $\omega_{3}=\omega^{\prime}$, и приведено представление в терминах эллиптических функций Вейерштрасса, тета-функций и эллиптических функций Якоби [10]. При подходяших интервалах изменения $z$ решения являются вешественными. Их вырождения дают все четные решения с одним лишь двойным полюсом при $x=0$ на вещественной оси. Эти вырождения могут совпадать с вырождениями решения Руджинарса-Шнайдера. Можно непосредственно проверить, что эти новые решения действительно удовлетворяют уравнению (4) при четных $n$ [11], однако для доказательства того, что таким образом исчерпываются все решения, пришлось развить новые методы.

Открытые Руджинарсом и Шнайдером модели не только демонстрируют действие алгебры Пуанкаре, но являются также полностью интегрируемыми. В частности, Руджинарс и Шнайдер показали пуассонову коммутативность для их решений переменных светового конуса

$$
S_{ \pm k}=\sum_{\substack{I \subseteq\{1,2, \ldots, n\} \\|I|=k}} \exp \left( \pm \sum_{i \in I} p_{i}\right) \prod_{\substack{i \in I \\ j \notin I}} f\left(x_{i}-x_{j}\right)
$$

так что $H=\left(S_{1}+S_{-1}\right) / 2$ и $P=\left(S_{1}-S_{-1}\right) / 2$. (Заметим, что четность или нечетность функций $f(x)$ означает, что в действительности имеется только $n$ функционально независимых величин.) Нерешенным остается вопрос о том, приводят ли новые решения из теоремы 3 к интегрируемым системам. Известно, что если использовать анзац Руджинарса и Шнайдера, то эти новые решения не всегда приводят к коммутирующим относительно скобки Пуассона величинам, однако в настоящий момент мы не можем исключить наличие других сохраняющихся величин, коммутирующих относительно скобки Пуассона [11].

Руджинарс [12] исследовал также квантовый вариант классических моделей, введенных им и Шнайдером. С самого начала он искал операторные аналоги переменных светового конуса (6). Он показал, что при $k=1, \ldots, n$

$$
\widehat{S}_{k}=\sum_{\substack{I \subseteq\{1,2, \ldots, n\} \\|I|=k}} \prod_{\substack{i \in I \\ j \notin I}} h\left(x_{j}-x_{i}\right)^{1 / 2} \exp \left(-\sqrt{-1} \beta \sum_{i \in I} \partial_{i}\right) \prod_{\substack{i \in I \\ j \notin I}} h\left(x_{i}-x_{j}\right)^{1 / 2}
$$

попарно коммутируют тогда и только тогда, когда уравнение (1) выполняется для всех $k$ и $n \geqslant 1$. Затем он показал, что выражение (2) дает решение уравнения $(1)$, и это решение связано с известным ранее решением Руджинарса-Шнайдера соотношением

$$
\frac{\sigma(x+\nu) \sigma(x-\nu)}{\sigma^{2}(x) \sigma^{2}(\nu)}=\wp(\nu)-\wp(x) .
$$

Руджинарс [12] предположил, что это решение "скорее всего единственно", но не смог этого доказать. Наш классический анализ дает возможные функции $F(x)=h(x) h(-x)$. Возникает естественный вопрос: имеется ли решение уравнения (1), соответствуюшее нашим новым решениям? Если нет, то решение Руджинарса действительно единственно. Теорема 1 доказывает единственность решений Руджинарса.

Теперь мы обратимся к доказательству теоремы 1, используя метод преобразования, развитьй в работе [9]. 


\section{2. ДОКАЗАТЕЛЬСТВО ТЕОРЕМЫ 1}

В этом разделе мы решим уравнение (1) путем построения преобразования Фурье этого уравнения при $k=1$. Наряду с функцией $F(z)=h(z) h(-z)$ введем функцию

$$
g(z, \beta)=h(-z) h(z-i \beta) .
$$

Преобразование Фурье уравнения (1) будет выполнено в терминах трансформанты $\Phi$ урье $\hat{g}(k, \beta)$ функции $g(z, \beta)$. Мы покажем, как после тшательного выбора параметризации преобразование Фурье уравнения (1) при $k=1$ приводит точно к тому же уравнению, которое возникло при исследовании преобразования Фурье уравнения (4). Тогда по теореме 3 получаем обшие решения для $g(z, \beta)$. Мы обнаружили, что можно записать

$$
g(z, \beta) \equiv h(-i \beta)\left(F_{1}(z-i \beta)-F_{1}(z)-F_{1}(\nu-i \beta)+F_{1}(\nu)\right),
$$

где $F_{1}(z)=\int F(z) d z, F(\nu)=0$, а $F(x)$ - любое решение, даваемое теоремой 3. Задав функцию $g(z, \beta)$, мы хотим теперь факторизовать ее, как указано в (7). Новые решения из теоремы 3 не обладают свойствами факторизации (7). Это означает, что при $k=1$ единственные решения уравнения (1) даются выражением (2). Однако известно, что эти решения удовлетворяют уравнению (1) для всех $k$, что и позволяет доказать теорему. Наша стратегия будет заключаться в том, чтобы брать преобразование Фурье для функций возрастаюшей сложности, в первую очередь рассматривая функции, имеющие только полюс в начале координат и обрашаюшиеся в нуль на бесконечности; затем мы рассмотрим аналогичные функции, стремяшиеся к постоянной на бесконечности; наконец, мы рассмотрим функции с периодическим набором полюсов вдоль вещественной оси, включая начало координат.

Преж де чем мы выведем уравнение для $\hat{g}$, рассмотрим свойства функции $g$ и условия, которые эти свойства налагают на $\hat{g}$. Исходные решения Руджинарса-Шнайдера для $F(z)$ можно выразить как

$$
F(z)=A \wp\left(z, g_{2}, g_{3}\right)+B
$$

где $A, B, g_{2}$ и $g_{3}$ - константы. Они являются четными функциями $z$ с двойным полюсом в начале координат. Новые решения уравнения, полученные в работе [9], имеют аналогичную структуру, но не позволяют добавить произвольную константу $B$. Однако в обоих случаях без потери общности можно использовать константу $A$ и скейлинговые свойства функции $\wp(z)$ для ограничения выбора $F(z)$ так, чтобы $z^{2} F(z) \rightarrow-1$ при $z \rightarrow 0$ и чтобы в случае, когда решение имеет конечный вешественный период, он полагался равным $2 \pi$. Пусть $\nu$ - нуль функции $F(z)$. Константу $B$ можно выразить через $\nu$ таким образом, чтобы решения (8) приняли вид

$$
F(z)=\wp(\nu)-\wp(z) .
$$

Условие $F(\nu)=0$ тогда требует, чтобы $h(\nu) h(-\nu)=0$. Фиксируем $h(z)$, потребовав, чтобы $h(z)=0$ при $z=-\nu$. Из этого следует $g(\nu, \beta)=g(-\nu+i \beta, \beta)=0$. Далее, поскольку $F(z)$ имеет двойной полюс при $z=0$, функция $h(z)$ должна иметь простой полюс при $z=0$, и мы выбираем $h(z)$ так, чтобы $z h(z) \rightarrow+1$ при $z \rightarrow 0$. Таким образом, функция $g(z, \beta)$ должна иметь простые полюсы при $z=0$ и $z=i \beta$, причем $z g(z) \rightarrow$ $-h(-i \beta)$ при $z \rightarrow 0$ и $(z-i \beta) g(z) \rightarrow h(-i \beta)$ при $z \rightarrow i \beta$. 
Получим теперь уравнение для $\hat{g}$, выполнив преобразование Фурье уравнения (1). Положим $z_{j}=x_{j}+i y_{j}$ и обозначим через $E\left(\mathbf{k}, z_{n}\right)$ левую часть уравнения (1) при $k=1$. Определим $(n-1)$-мерное преобразование Фурье как

$$
\widehat{E}\left(\mathbf{k}, z_{n}, \beta\right)=\int_{\mathbb{R}^{n-1}} E\left(\mathbf{z}, z_{n}, \beta\right) e^{-i \mathbf{k} \mathbf{z}} d \mathbf{z}
$$

где $\mathbf{z}$ - вектор $\left(z_{1}, z_{2}, \ldots, z_{n-1}\right)$. Однако поскольку $g(z, \beta)$ имеет полюс в начале координат, мы заменим $z_{j}$ на $z_{j}+i \epsilon_{j}$ и будем считать, что $\epsilon_{1}>\epsilon_{2}>\cdots>\epsilon_{n}>0$ и $\epsilon_{1}$ мало. В определении $\widehat{E}(10)$ предполагается, что интегрирование проводится вдоль вешественной оси в комплексной плоскости $x_{j}+i y_{j}$.

2.1. Функции с бесконечным вещественным периодом, обращающиеся в нуль на бесконечности. В первую очередь рассмотрим класс решений $g(z, \beta)$, которые имеют бесконечный вешественный период и стремятся к нулю на бесконечности, не имея при этом никаких других сингулярностей на вещественной оси, кроме полюса в начале координат. Тем самым при замене $z_{j}$ на $z_{j}+i \epsilon_{j}$ подынтегральное выражение в (10) не будет иметь других сингулярностей в области интегрирования, если $\epsilon_{1}<\beta$, где $\beta$ вешественна и положительна. Редукция $\widehat{E}=0$ при $\epsilon_{1} \rightarrow 0$ к уравнению, включающему обобщенный фурье-образ $\hat{g}(k, \beta)$, следует подходу, предложенному в работе [9]. Определение $\hat{g}$ имеет вид

$$
\hat{g}(k, \beta)=\frac{1}{2}\left(\hat{g}_{\mathrm{U}}(k, \beta)+\hat{g}_{\mathrm{L}}(k, \beta)\right),
$$

где

$$
\hat{g}_{\mathrm{U}}(k, \beta)=f_{-\infty}^{\infty} g(z, \beta) e^{-i k z} d z, \quad \hat{g}_{\mathrm{L}}(k, \beta)=f_{-\infty}^{\infty} g(z, \beta) e^{-i k z} d z
$$

определены так, что пути интегрирования проходят соответственно над и под полюсом при $z=0$. Имеем тогда

$$
\hat{g}_{\mathrm{L}}-\hat{g}_{\mathrm{U}}=2 \pi i \times\left.\operatorname{Res}\right|_{z=0} g(z, \beta)=-2 \pi i h(-i \beta)
$$

И

$$
\hat{g}_{\mathrm{U}}=\hat{g}+i \pi h(-i \beta), \quad \hat{g}_{\mathrm{L}}=\hat{g}-i \pi h(-i \beta) .
$$

Однако приведенное выше определение сопряжено с проблемой при $\beta \rightarrow 0$, заключающейся в том, что

$$
\lim _{\beta \rightarrow 0} \hat{g}(k, \beta) \neq \hat{g}(k, 0) \text {. }
$$

Это связано с тем, что при $\beta>0$ оба пути интегрирования в (12) лежат ниже полюса при $z=i \beta$, тогда как если мы положим $\beta=0$ и затем используем определения (11) и (12), то верхний путь интегрирования пройдет выше полюса, которьй становится двойным полюсом в точке $z=0$ при $\beta \rightarrow 0$. Чтобы преодолеть эту трудность, определим 
модифицированную функцию $\hat{g}_{\mathrm{U}}$, обозначаемую $\hat{g}_{\mathrm{UM}}$, и модифицированный обобшенный фурье-образ $\hat{g}_{\mathrm{M}}$, сдвигая верхний контур в уравнении (12) таким образом, чтобы он проходил над полюсом при $z=i \beta$. Тогда

$$
\hat{g}_{\mathrm{UM}}=\hat{g}_{\mathrm{U}}-\left.2 \pi i \operatorname{Res}\right|_{z=i \beta} g(z, \beta) e^{-i k z}=\hat{g}_{\mathrm{U}}-2 \pi i h(-i \beta) e^{\beta k} .
$$

Отсюда получаем

$$
\hat{g}_{\mathrm{M}}=\hat{g}-\pi i h(-i \beta) e^{\beta k} .
$$

С помошью этих определений легко показать, что когда $g=-1 /(z(z-i \beta))$, имеем $\hat{g}_{\mathrm{M}}=\pi\left(e^{\beta k}-1\right)(\operatorname{sign} k) / \beta$ при $\lim _{\beta \rightarrow 0} \hat{g}_{\mathrm{M}}=\pi|k|$, что представляет собой обобшенное преобразование Фурье функции $-1 / z^{2}$.

В результате преобразования Фурье уравнения (1) при $k=1$ каждый из членов

$$
\prod_{j \neq i} h\left(x_{j}-x_{i}\right) h\left(x_{i}-x_{j}-i \beta\right)=\prod_{j \neq i} g\left(x_{i}-x_{j}\right)
$$

в сумме редуцируется в произведение одномерных фурье-образов. Используя приведенные выше определения, получаем, что в зависимости от положения полюсов возникает одна из четырех возможностей:

$$
\begin{gathered}
\int_{-\infty}^{\infty} g(u+i \epsilon) e^{-i k u} d u=\hat{g}_{\mathrm{M}}(k)+i \pi h(-i \beta)-i \pi h(-i \beta) e^{\beta k}, \\
\int_{-\infty}^{\infty} g(u-i \epsilon) e^{-i k u} d u=\hat{g}_{\mathrm{M}}(k)-i \pi h(-i \beta)+i \pi h(-i \beta) e^{\beta k}, \\
\int_{-\infty}^{\infty} g(-u+i \epsilon) e^{-i k u} d u=\hat{g}_{\mathrm{M}}(k)+i \pi h(-i \beta)-i \pi h(-i \beta) e^{-\beta k}, \\
\int_{-\infty}^{\infty} g(-u-i \epsilon) e^{-i k u} d u=\hat{g}_{\mathrm{M}}(k)-i \pi h(-i \beta)+i \pi h(-i \beta) e^{-\beta k}
\end{gathered}
$$

Так, например, при $n=3$

$$
\begin{aligned}
g\left(z_{1}-\widehat{\left.z_{2}\right) g\left(z_{1}\right.}-z_{3}\right)= & \int_{\mathbb{R}^{2}} g\left(z_{1}-z_{2}+i\left(\epsilon_{1}-\epsilon_{2}\right)\right) \times \\
& \times g\left(z_{1}-z_{3}+i\left(\epsilon_{1}-\epsilon_{3}\right)\right) e^{-i k_{1} z_{1}-i k_{2} z_{2}} d z_{1} d z_{2}= \\
= & e^{-i\left(k_{1}+k_{2}\right) z_{3}} \int_{-\infty}^{\infty} d v g\left(v+i \epsilon^{\prime}\right) e^{-i\left(k_{1}+k_{2}\right) v} \times \\
& \times \int_{-\infty}^{\infty} d u g\left(-u+i \epsilon^{\prime \prime}\right) e^{-i k_{2} u}= \\
= & e^{-i\left(k_{1}+k_{2}\right) z_{3}}\left[\hat{g}_{\mathrm{M}}\left(k_{1}+k_{2}\right)+i \pi h(-i \beta)-i \pi h(-i \beta) e^{\beta\left(k_{1}+k_{2}\right)}\right] \times \\
& \times\left[\hat{g}_{\mathrm{M}}\left(-k_{2}\right)+i \pi h(-i \beta)-i \pi h(-i \beta) e^{-\beta k_{2}}\right] .
\end{aligned}
$$

Здесь мы положили $u=z_{2}-z_{1}, v=z_{1}-z_{3}, \epsilon^{\prime}=\epsilon_{1}-\epsilon_{3}$ и $\epsilon^{\prime \prime}=\epsilon_{1}-\epsilon_{2}$. Наличие обшего множителя $h(-i \beta)$ в этих выражениях подсказывает записать $\hat{g}_{\mathrm{M}}(k, \beta)$ в виде 
$\hat{g}_{\mathrm{M}}(k, \beta)=-i I(k, \beta) \beta h(-i \beta)$. Тогда в пределе $\beta \rightarrow 0$ имеем $\hat{g}_{\mathrm{M}}(k, 0)=I(k, 0)$. Используя это, находим, что равенство (10) при $n=3$ можно записать как $\sum_{j=1}^{3} J_{j}=0$, где, например,

$$
\begin{aligned}
J_{1}= & -\left(I\left(k_{1}+k_{2}, \beta\right) \frac{\beta}{\pi}-1+e^{\beta\left(k_{1}+k_{2}\right)}\right)\left(I\left(-k_{2}, \beta\right) \frac{\beta}{\pi}-1+e^{-\beta k_{2}}\right)+ \\
& +\left(I\left(-k_{1}-k_{2}, \beta\right) \frac{\beta}{\pi}+1-e^{-\beta\left(k_{1}+k_{2}\right)}\right)\left(I\left(k_{2}, \beta\right) \frac{\beta}{\pi}+1-e^{\beta k_{2}}\right)
\end{aligned}
$$

что соответствует $g\left(z_{1}-\widehat{\left.z_{2}\right) g(} z_{1}-z_{3}\right)-g\left(z_{2}-\widehat{\left.z_{1}\right) g(} z_{3}-z_{1}\right)$ в сумме, полученной из $(1)$, и имеются аналогичные определения для $J_{2}$ и $J_{3}$ :

$$
\begin{aligned}
J_{2}= & \left(I\left(-k_{1}-k_{2}, \beta\right) \frac{\beta}{\pi}+1-e^{-\beta\left(k_{1}+k_{2}\right)}\right)\left(I\left(k_{1}, \beta\right) \frac{\beta}{\pi}-1+e^{\beta k_{1}}\right)- \\
& -\left(I\left(k_{1}+k_{2}, \beta\right) \frac{\beta}{\pi}-1+e^{\beta\left(k_{1}+k_{2}\right)}\right)\left(I\left(-k_{1}, \beta\right) \frac{\beta}{\pi}+1-e^{-\beta k_{1}}\right), \\
J_{3}= & \left(I\left(k_{1}, \beta\right) \frac{\beta}{\pi}-1+e^{\beta k_{1}}\right)\left(I\left(k_{2}, \beta\right) \frac{\beta}{\pi}-1+e^{\beta k_{2}}\right)- \\
& -\left(I\left(-k_{1}, \beta\right) \frac{\beta}{\pi}+1-e^{-\beta k_{1}}\right)\left(I\left(-k_{2}, \beta\right) \frac{\beta}{\pi}+1-e^{-\beta k_{2}}\right) .
\end{aligned}
$$

Введем функцию $\widehat{G}(k, \beta)$ так, что в ретроспективе это окажется предусмотрительной заменой переменной:

$$
I(k, \beta)=\widehat{G}(k, \beta) \frac{e^{\beta k}-1}{k \beta} .
$$

Эту замену переменной подсказывает внимательное изучение ряда

$$
I(k, \beta)=\sum_{j=0}^{\infty} I_{j}(k) \beta^{j}
$$

возникаюшего из $\sum_{j=1}^{3} J_{j}=0$ с использованием приведенных выше выражений. Как бы то ни было, $I \rightarrow \widehat{G}(k, 0)$ при $\beta \rightarrow 0$, и нам известно (поскольку $F(x)$ четна), что $\widehat{G}(k, 0)$ является четной. Рассмотрение уравнения для $\widehat{G}(k, \beta)$ (например, разложением в ряд) показывает, что $\widehat{G}(k, \beta)$ сама является четной. Этот факт приводит к упрошениям. В случае четности $\widehat{G}(k, \beta)$ выражение для $J_{1}$ упрошается:

$J_{1}=-\frac{\left(e^{\beta k_{1}}-1\right)\left(e^{\beta k}-1\right)\left(1-e^{-\beta\left(k_{1}+k_{2}\right)}\right)}{\left(k_{1}+k_{2}\right) k_{2}}\left(\widehat{G}\left(k_{2}, \beta\right)-\pi k_{2}\right)\left(\widehat{G}\left(k_{1}+k_{2}, \beta\right)+\pi\left(k_{1}+k_{2}\right)\right)$,

и аналогично для $J_{2}, J_{3}$. Далее находим, что содержащие $\beta$ экспоненциальные множители являются обшими для всех $J_{i}$, и тогда равенство (10) для случая $n=3$ можно окончательно записать как

$$
\left(k_{2} \widehat{G}\left(k_{1}, \beta\right)+k_{1} \widehat{G}\left(k_{2}, \beta\right)\right) \widehat{G}\left(k_{1}+k_{2}, \beta\right)-\left(k_{1}+k_{2}\right) \widehat{G}\left(k_{1}, \beta\right) \widehat{G}\left(k_{2}, \beta\right)=\pi^{2} k_{1} k_{2}\left(k_{1}+k_{2}\right) .
$$


Теперь это уравнение представляет собой в точности уравнение для фурье-образа $F(z)$, полученное в работе [9] (см. уравнение (5.5) в указанной работе) при исследовании уравнения (4) при $n=3$. Единственными решениями требуемого вида являются функция $\widehat{G}(k, \beta) \equiv \widehat{G}(k)=\widehat{F}(k)=\pi k \operatorname{cth}(\pi k / a)$ и ее предел при $a \rightarrow 0$, а именно $\pi|k|$. Отсюда

$$
\hat{g}_{\mathrm{M}}(k, \beta)=-i \beta h(-i \beta) \frac{e^{\beta k}-1}{\beta k} \widehat{F}(k),
$$

или

$$
g(z, \beta)=h(-i \beta)\left(\int F(z-i \beta) d z-\int F(z) d z\right) .
$$

Легко проверить, что при

$$
\widehat{F}(k)=\pi k \operatorname{cth}\left(\frac{\pi k}{a}\right), \quad F(z)=-\frac{1}{4} \frac{a^{2}}{\operatorname{sh}^{2}(a z / 2)}
$$

имеем

$$
\begin{aligned}
g(z, \beta) & =\frac{a}{2} h(-i \beta)\left(\operatorname{cth}\left(\frac{1}{2} a(z-i \beta)\right)-\operatorname{cth}\left(\frac{1}{2} a z\right)\right)= \\
& =\frac{a}{2} h(-i \beta) \frac{\operatorname{sh}(i a \beta / 2)}{\operatorname{sh}(a z / 2) \operatorname{sh}(a(z-i \beta) / 2)} \equiv h(-z) h(z-i \beta)
\end{aligned}
$$

где $h(z)=a /(2 \operatorname{sh}(a z / 2))$, что дает соответствующую факторизацию $F(z)=h(-z) h(z)$.

Подобным же образом (или при $a \rightarrow 0$ ) для $\widehat{F}(k)=\pi|k|$ и $F(z)=-1 / z^{2}$ имеем

$$
g(z, \beta)=h(-i \beta)\left(\frac{1}{z-i \beta}-\frac{1}{z}\right)=h(-i \beta) \frac{-i \beta}{(-z)(z-i \beta)} \equiv h(-z) h(z-i \beta),
$$

где $h(z)=1 / z$ и $F(z)=h(-z) h(z)$. Обе эти факторизации определены с точностью до сдвига на экспоненту $h(z) \rightarrow h(z) e^{\alpha z}$, заданную в формуле (2).

Для случая $n \geqslant 4$ находим также, что тот же набор преобразований $\hat{g}_{\text {м редуцирует }}$ формулу (10) к исходному выражению для фурье-образа $\widehat{F}$, возникающего при исследовании уравнения (4). При нечетных $n$ имеются только решения (19) и (22), тогда как при четных $n$, кроме этих решений, имеется еше и решение

$$
\widehat{F}(k)=\pi k \operatorname{th}\left(\frac{\pi k}{2 a}\right), \quad F(z)=-\frac{a^{2} \operatorname{ch} a z}{\operatorname{sh}^{2} a z} .
$$

Теперь, однако, мы имеем

$$
g(z, \beta)=a h(-i \beta)\left(\frac{1}{\operatorname{sh}(a(z-i \beta))}-\frac{1}{\operatorname{sh} a z}\right),
$$

а это выражение нельзя записать в виде $g(z, \beta)=h(-z) h(z-i \beta)$. Следовательно, отсюда нельзя получить решений уравнения (1). 
2.2. Функции с бесконечным вещественным периодом, постоянные на бесконечности. Решения Руджинарса-Шнайдера (8) содержат дополнительную произвольную константу, которая в гиперболическом пределе соответствует функции, не обращающейся в нуль на бесконечности. Новые решения из работы [9], примером которых является решение (23), не имеют этой степени свободы: все гиперболические вырождения этих решений стремятся к нулю на бесконечности. Чтобы исследовать функции, которые не стремятся к нулю на бесконечности, нам приходится иметь дело с преобразованиями Фурье обобщенных функций. Добавление константы к $F(z)$ требует добавления аналогичной константы к $g(z, \beta)$, поскольку, например, если $F \rightarrow a^{2}$ при $z \rightarrow \infty$, то $g \rightarrow a^{2}$ при $z \rightarrow \infty$. При $n=3$ легко проверить, что преобразование $g(z, \beta) \rightarrow g(z, \beta)+$ const оставляет уравнение (1) неизменным. Однако это не выполняется автоматически в случае $n>3$. Мы нашли, что при $n>3$ преобразование $g(z, \beta) \rightarrow g(z, \beta)+A$ требует дополнительных условий на $g$, чтобы уравнение было инвариантно относительно этого преобразования. При $n=4$ имеется только одно дополнительное условие, которое автоматически выполняется для решения $g$, соответствуюшего решению Руджинарса-Шнайдера, однако не для решения, соответствуюшего новым решениям [9]. Мы полагаем, что при $n>4$ решение Руджинарса-Шнайдера автоматически удовлетворяет всем дополнительным условиям, а даваемые теоремой 3 новые решения нет. Добавление произвольной константы $A$ к $g$ требует добавления $A \delta(k)$ к $\hat{g}(k, \beta)$. Для решений $\hat{g}$, которые во всех других отношениях представляют собой функции от $k$ с "хорошим" поведением, легко получить решение для $g(z, \beta)$ и соответствуюшее решение для $h(z)$, когда они сушествуют. Например, если изменить функцию $F(z)$ в $(20)$, добавив к ней произвольную константу, и потребовать, чтобы $F(\nu)=0$, то получим

$$
F(z)=\frac{1}{4} a^{2}\left\{\frac{1}{\operatorname{sh}^{2}(a \nu / 2)}-\frac{1}{\operatorname{sh}^{2}(a z / 2)}\right\},
$$

так что

$$
g(z, \beta)=\frac{1}{2} a h(-i \beta) \frac{\operatorname{sh}(-a i \beta / 2)}{\operatorname{sh}(-a z / 2) \operatorname{sh}(a(z-i \beta) / 2)}+A .
$$

Поскольку по определению $g(z, \beta)=h(-z) h(z-i \beta)$ и функция $h(z)$ имеет нуль при $z=-\nu$, имеем $g(\nu, \beta)=0$, как было указано ранее. Отсюда получаем

$$
A \equiv A(\beta, \nu)=-\frac{1}{2} a h(-i \beta) \frac{\operatorname{sh}(-a i \beta / 2)}{\operatorname{sh}(-a \nu / 2) \operatorname{sh}(a(\nu-i \beta) / 2)} .
$$

Теперь можно выразить $g$ как

$$
\begin{aligned}
g(z, \beta) & =\frac{1}{2} a h(-i \beta) \frac{\operatorname{sh}(a(-z+\nu) / 2)}{\operatorname{sh}(a \nu / 2) \operatorname{sh}(-a z / 2)} \frac{\operatorname{sh}(a(z+\nu-i \beta) / 2)}{\operatorname{sh}(a(z-i \beta) / 2)} \frac{\operatorname{sh}(-a i \beta / 2)}{\operatorname{sh}(a(\nu-i \beta) / 2)} \equiv \\
& \equiv h(-z) h(z-i \beta),
\end{aligned}
$$

где

$$
h(z)=\frac{1}{2} a \frac{\operatorname{sh}(a(z+\nu) / 2)}{\operatorname{sh}(a z / 2) \operatorname{sh}(a \nu / 2)}
$$


при

$$
h(-z) h(z)=\frac{a^{2}}{4} \frac{\operatorname{sh}(a(z-\nu) / 2) \operatorname{sh}(a(z+\nu) / 2)}{\operatorname{sh}^{2}(a z / 2) \operatorname{sh}^{2}(a \nu / 2)}=\frac{a^{2}}{4}\left\{\frac{1}{\operatorname{sh}^{2}(a \nu / 2)}-\frac{1}{\operatorname{sh}^{2}(a z / 2)}\right\},
$$

как и требовалось. Заметим также, что при $\nu \rightarrow \infty$ получаем, что $h(z)$ стремится к решениям из п. 2.1, умноженным на экспоненциальный множитель $e^{a z / 2}$, и наша факторизация однозначна только с точностью до таких членов.

Аналогичное, но более простое вычисление для $F(z)=1 / \nu^{2}-1 / z^{2}$ дает

$$
g(z, \beta)=h(-i \beta) \frac{\nu(-i \beta)}{\nu-i \beta} \frac{(-z+\nu)}{(-z) \nu} \frac{z+\nu-i \beta}{(z-i \beta) \nu},
$$

так что $h(z)=(z+\nu) /(z \nu)$ при $h(z) h(-z)=1 / \nu^{2}-1 / z^{2}$.

2.3. Периодические функции. Для $2 \pi$-периодических функций, которые имеют периодический набор двойных полюсов при $z=2 \pi p$, мы показали [9], что подходящим видом преобразования $\hat{g}(k, \beta)$ является $\sum_{p=-\infty}^{\infty} a_{p} \delta(k-p)$, что соответствует ряду $\Phi$ рье $(1 / 2 \pi) \sum_{p=-\infty}^{\infty} a_{p} e^{i p z}$ для $g(z, \beta)$. Уравнение, которому удовлетворяет $\hat{g}$, такое же, как и в непериодическом случае, однако оно решается только для целочисленных значений $\left\{k_{j}\right\}$. Таким образом, если $\hat{g}(k, \beta)$ является решением в непрерывном случае, имеем решение $a_{p}(\beta)=\hat{g}(p, \beta)$. Соответствуюшее решение $g_{2 \pi}(z, \beta)$ является периодическим продолжением непрерывного случая, выраженным как

$$
g_{2 \pi}(z, \beta)=\sum_{p=-\infty}^{\infty} g(z-2 \pi p)
$$

где $g(z)$ - непериодическое решение, определяемое равенством (19) с конкретными выражениями $(21),(22),(24),(28)$ и (31).

Для функции $F(z)=1 / \mathrm{sh}^{2} z$ это соответствует ю-функции. Поскольку без потери общности мы положили, что для $F(z)$ выполнено $z^{2} F(z)=-1$ при $z \rightarrow 0$, то можно использовать скейлинговые свойства функции $\wp\left(z, g_{2}, g_{3}\right)=a^{2} \wp\left(a z, g_{2} / a^{4}, g_{3} / a^{6}\right)$, для того чтобы положить $F(z)=-\wp(z)$. Добавление константы к функции $F$ приводит к добавлению константы к $g$, так что

$$
F \rightarrow F+B \Rightarrow \int F \rightarrow \int F+B z+B_{1} \Rightarrow g \rightarrow g-i B \beta h(-i \beta) .
$$

Отсюда следует, что соответствуюшее решению (21) периодическое решение есть

$$
g(z, \beta)=-h(-i \beta)(\zeta(z)-\zeta(z-i \beta)+C(\beta)),
$$

где $C(\beta)$-произвольная функция от $\beta$, зависяшая также от параметра $\nu$ и определяемая условием $g(\nu, \beta)=0$. Следовательно,

$$
\begin{aligned}
g(z, \beta) & =-h(-i \beta)(\zeta(z)-\zeta(z-i \beta)-\zeta(\nu)+\zeta(\nu-i \beta))= \\
& =h(-i \beta) \frac{\sigma(\nu) \sigma(-i \beta)}{\sigma(\nu-i \beta)} \frac{\sigma(-z+\nu)}{\sigma(-z) \sigma(\nu)} \frac{\sigma(z+\nu-i \beta)}{\sigma(z-i \beta) \sigma(\nu)} \equiv h(-z) h(z-i \beta)
\end{aligned}
$$


при $h(z)=\sigma(z)=\sigma(z+\nu) /(\sigma(z) \sigma(\nu))$.

Выражение для $g$ в случае новых решений Байатт-Смита и Брадена [9] можно записать в виде, аналогичном (34), однако оно снова не может быть факторизовано в произведение $h(-z) h(z-i \beta)$. Например, рассмотрим функцию $F(z)=-\operatorname{cn} z \operatorname{dn} z / \operatorname{sn}^{2} z$, для которой справедливо равенство $\int F(z) d z=1 / \operatorname{sn} z$. Тогда из (19) имеем

$$
g(z, \beta)=h(-i \beta)\left(\frac{1}{\operatorname{sn}(z-i \beta)}-\frac{1}{\operatorname{sn} z}\right),
$$

и разложение в ряды показывает, что это выражение нельзя записать в факторизованном виде.

2.4. Другое функциональное уравнение. Мы доказали теорему 1. Мы показали, что преобразование Фурье уравнения (1) при $k=1$ приводит к уравнению

$$
g(z, \beta) \equiv h(-i \beta)\left(F_{1}(z-i \beta)-F_{1}(z)+C(\beta)\right)=h(-z) h(z-i \beta) .
$$

Здесь $F_{1}(z)=\int F(z) d z$ и $C(\beta)=-F_{1}(\nu-i \beta)+F_{1}(\nu)$, поскольку мы положили, что $g(\nu, \beta)=0$. Мы нашли, что $F(z)$ должно являться решением, даваемым теоремой 3, и что среди таких решений только те решения, которые задаются формулой (2), допускают желаемую факторизацию. В заключение покажем, что анализ уравнения (37) непосредственно приводит к этому результату.

Рассмотрим уравнение (37) как функциональное уравнение для $C, h$ и $F_{1}$ и в соответствии с этим определим $g$. Решим его при подходящих условиях, которые сохраняются от исходной задачи:

$$
z h(z) \rightarrow a, \quad z F_{1}(z) \rightarrow a, \quad g(\nu, \beta)=0 .
$$

Последнее условие означает, что $C(\beta)=-F_{1}(\nu-i \beta)+F_{1}(\nu)$, и, следовательно, $C(0)=0$. Заметим, что уравнение (37) инвариантно по отношению к преобразованию

$$
F_{1}(z) \rightarrow F_{1}(z)+B z+B_{1}, \quad C(\beta) \rightarrow C(\beta)+i \beta B .
$$

Этот произвол можно фиксировать, потребовав в дальнейшем, чтобы $C(0)=C^{\prime}(0)=0$.

Чтобы решить уравнение (37), разложим его в степенной ряд по $\beta$. Первые нетривиальные члены дают уравнения, достаточные для исключения $h(z)$ и $h(-z)$ и для вывода уравнения третьего порядка на $F_{1}(z)$ в терминах коэффициентов $C_{0}, C_{1}, b_{0}, b_{1}, b_{2}$ в разложениях

$$
C(\beta)=\sum_{j=0}^{\infty} C_{j} \beta^{j+2}, \quad h(-i \beta)=\sum_{j=0}^{\infty} b_{j}(-i \beta)^{j-1} .
$$

Получающееся уравнение третьего порядка на $F_{1}$

$$
\left(2 F_{1}^{\prime \prime \prime}(x) F_{1}^{\prime}(x)-3 F_{1}^{\prime \prime}(x)^{2}\right) b_{0}^{2}+12 C_{0}^{2} b_{0}^{2}+12 F_{1}^{\prime}(x)^{2}\left(b_{1}^{2}-2 b_{2} b_{0}\right)+24 i C_{1} b_{0}^{2} F_{1}^{\prime}(x)=0
$$


можно проинтегрировать, что дает

$$
F_{1}(z)=b_{0} \zeta\left(z, g_{2}, g_{3}\right)+z b_{0} \wp\left(\nu, g_{2}, g_{3}\right)
$$

при этом $C$ определяется как

$$
C(\beta)=b_{0} \zeta(\nu)-b_{0} \zeta(\nu-i \beta)+i \beta b_{0} \wp(\nu)
$$

(остаюшиеся константы в этом выражении определяются ниже в терминах $C_{0}, C_{1}, b_{1}$ и $\left.b_{2}\right)$. Функция $h(z)$ тогда определяется уравнением

$$
\frac{h^{\prime}}{h}=\frac{1}{2} \frac{F_{1}^{\prime \prime}}{F_{1}^{\prime}}+\frac{1}{2} b_{0} \wp^{\prime}(\nu)+\frac{b_{1}}{b_{0}}
$$

с решением

$$
h(z)=b_{0} \frac{\sigma(z+\nu)}{\sigma(z) \sigma(\nu)} e^{\alpha z}
$$

Четыре константы $C_{0}, C_{1}, b_{1}, b_{2}$ определяют четыре константы, появляющиеся в уравнениях (41), (42), а именно $\wp(\nu), \xi(\nu), g_{2}$ и $\wp^{\prime}(\nu)$, причем $g_{3}$ задается с помощью $\wp(\nu), \wp^{\prime}(\nu)$ и $g_{2}$. Разумеется, чтобы удовлетворить условиям $(38)$, мы также потребуем, чтобы $b_{0}=a$. Соотношения между двумя наборами констант имеют вид

$$
\begin{aligned}
C_{0} & =-\frac{1}{2} b_{0} \wp^{\prime}(\nu), & C_{1} & =i b_{0}\left(\wp(\nu)-\frac{1}{6} g_{2}\right) \\
b_{1} & =b_{0}(\zeta(\nu)+\alpha), & b_{2} & =\frac{1}{2} b_{0}\left((\zeta(\nu)+\alpha)^{2}-\wp(\nu)\right) .
\end{aligned}
$$

Константа $\alpha$ произвольна и не влияет на решение (41), поскольку ясно, что отношение $h(-z) h(z-i \beta) / h(-i \beta)$ не зависит от $\alpha$. Это является отмеченной ранее неоднозначностью факторизации.

В заключение отметим, что только решения Руджинарса-Шнайдера (2) приводят к решениям уравнения (37) и, следовательно, к решениям уравнения (1). Таким образом, теорема 1 снова доказана.

Благодарности. Мы благодарны А. М. Дейви и С. Н. М. Руджинарсу за полезные обсуждения. Один из авторов (Х.У.Б.) признателен Институту математических наук Исаака Ньютона (Кембридж, Великобритания) за поддержку во время выполнения данной работы. 


\section{Список литературы}

[1] S. N. M. Ruijsenaars, H. Schneider. Ann. Phys. 1986. V. 170. P. 370-405.

[2] F. Calogero. Lett. Nuovo Cimento. 1975. V. 13. P. 507-511; E. Gutkin. J. Math. Anal. Appl. 1988. V. 133. P. 122-134; B. Sutherland. Phys. Rev. Lett. 1975. V. 34. P. 1083-1085.

[3] H. W. Braden. J. Phys. A. 2001. V. 34. P. 2197-2204.

[4] Calogero-Moser-Sutherland Models. CRM Series in Mathematical Physics. Eds. J. F. van Diejen, L. Vinet. New York: Springer, 2000.

[5] H. W. Braden, R. Sasaki. Progr. Theor. Phys. 1997. V. 97. P. 1003-1018.

[6] H. W. Braden, V. M. Buchstaber. Reviews in Mathematics and Mathematical Physics. 1997. V. 10. P. 121-166; SIAM J. Math. Anal. 1997. V. 28. P. 903-923; Integrability: The Seiberg-Witten and Whitham Equations. Eds. H. W. Braden, I. M. Krichever. Amsterdam: Gordon and Breach, 2000; M. Bruschi, F. Calogero. SIAM J. Math. Anal. 1990. V. 21. Р. 1019-1030; В. М. Бухитабер, И. М. Кричевер. ТМФ. 1993. Т. 94. № 2. С. 200-212; F. Calogero. Lett. Nuovo Cimento. 1976. V. 16. P. 77-80; B. A. Dubrovin, A. S. Fokas, P. M. Santini. Duke Math. J. 1994. V. 76. P. 645-668; Б. М. Гуревич. Вестн. МГУ, Сер. I. Матем. Мех. 1987. Т. 112. С. $73-75$; УМН. 1990. Т. 45. С. 131-132; Asymptotically additive integrals of motion for particles with nonpairwise interaction in dimension one. In: Probability Contributions to Statistical Mechanics. Adv. Soviet Math. V. 20. Eds. H. W. Braden, I. M. Krichever. Providence, RI: Am. Math. Soc., 1994. P. 221-275; Б. М. Гуревич, О. В. Радкевич. УМН. 1994. Т. 49. С. 207-208; V. I. Inozemtsev. Lett. Math. Phys. 1989. V. 17. P. 11-17; Commun. Math. Phys. 1989. V. 121. P. 629-638.

[7] V. M. Buchstaber, A. M. Perelomov. Am. Math. Soc. Transl. Ser. 2. 1996. V. 175. P. 15-34.

[8] H. W. Braden, J. G. B. Byatt-Smith. Bull. London Math. Soc. 1999. V. 31. P. 463-470.

[9] J. G. B. Byatt-Smith, H. W. Braden. Functional equations and Poincaré invariant mechanical systems. Preprint EMPG-01-12. Edinburgh: EMPG, 2001; math-ph/0110012.

[10] E. T. Whittaker, G. N. Watson. A Course of Modern Analysis. Cambridge: Cambridge Univ. Press, 1927.

[11] H. W. Braden, J. G. B. Byatt-Smith. Phys. Lett. A. 2002. V. 295. P. 208-216; nlin.SI/0110014.

[12] S. N. M. Ruijsenaars. Commun. Math. Phys. 1987. V. 110. P. 191-213. 